INPLASY

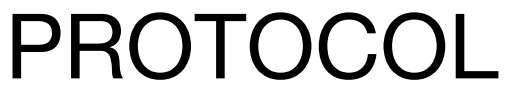

To cite: Ju et al. Predictors of mortality in patients with Methicillin-resistant

Staphylococcus aureus bloodstream infection: A metaanalysis. Inplasy protocol 202120082. doi:

10.37766/inplasy2021.2.0082

Received: 28 February 2021

Published: 28 February 2021

Corresponding author:

Mohan Ju

08301010203@fudan.edu.cn

Author Affiliation:

Huashan Hospital North,

Fudan University

Support: Shanghai Health

Commission.

Review Stage at time of this submission: Data analysis.

Conflicts of interest:

None declared.

\section{Predictors of mortality in patients with Methicillin-resistant Staphylococcus aureus bloodstream infection: A meta-analysis}

Ju, M1; Huang, Y2; Xu, X33 Qian, X4; Bi, Y3; Liu, S4; Li, X5; Dong, S6; Yuan, $\mathrm{J}^{7}$; Lin, $\mathrm{D}^{8}$.

Review question / Objective: What risk factors are associated with mortality in patients with Methicillin-resistant Staphylococcus aureus bloodstream infection?

Condition being studied: Some previous researches reported that patients with Methicillin-resistant Staphylococcus aureus bloodstream infection have higher mortality rate. Identification of risk factors for death caused by MRSA BSI is crucial for early implementation of an appropriate therapy and improving patients' outcome. Many studies have attempted to identify all risk factors, However, studies investigating predictors of mortality in patients with MRSA BSI produced inconsistent results. Therefore, we conducted a systematic review and meta-analysis to evaluate the relation between possible risk factors and mortality in patients with MRSA bloodstream infection.

INPLASY registration number: This protocol was registered with the International Platform of Registered Systematic Review and Meta-Analysis Protocols (INPLASY) on 28 February 2021 and was last updated on 28 February 2021 (registration number INPLASY202120082).

\section{INTRODUCTION}

Review question / Objective: What risk factors are associated with mortality in patients with Methicillin-resistant Staphylococcus aureus bloodstream infection?

Condition being studied: Some previous researches reported that patients with
Methicillin-resistant Staphylococcus aureus bloodstream infection have higher mortality rate. Identification of risk factors for death caused by MRSA BSI is crucial for early implementation of an appropriate therapy and improving patients' outcome. Many studies have attempted to identify all risk factors, However, studies investigating predictors of mortality in patients with MRSA BSI produced inconsistent results. 
Therefore, we conducted a systematic review and meta-analysis to evaluate the relation between possible risk factors and mortality in patients with MRSA bloodstream infection.

\section{METHODS}

Participant or population: Adult patients with MRSA bloodstream infection.

Intervention: Risk factors associated with mortality in patients with MRSA bloodstream infection.

Comparator: Survivors of patients with MRSA bloodstream infection.

Study designs to be included: Studies reporting risk factors of mortality in patients with MRSA bloodstream infection were included in this meta-analysis.

Eligibility criteria: In this meta-analysis, observational studies reporting mortality and associated risk factors of patients with MRSA bloodstream infection were included. The primary outcome was mortality. On the other hand, after reviewing by 2 independent examiners, non-original articles, such as reviews, meta-analyses, etc., case reports, in vitro or experimental animal studies, or pediatric researches were not included. Researches in which MRSA status ( infection/ colonization) was not clarified were also excluded.

Information sources: Pubmed, Embase and Cochrane library.

Main outcome(s): Mortality of patients with MRSA bloodstream infection.

Quality assessment / Risk of bias analysis: The Newcastle-Ottawa Scale score (NOS), ranging from 0-9 for quality assurance, was used for the methodological quality assessment of articles included. Two independent examiners (MH.J. and YY.H.) performed NOS for each study. Inconsistencies between the two investigators were extensively discussed until agreement was achieved. Studies with
NOS score of 5 or greater underwent further analysis, while others were excluded because of the potential high risk of bias.

Strategy of data synthesis: Statistical analyses were performed using Review Manager (version 5.3 software). We tested between study heterogeneity with the $Q$ statistic (significant when $p<0.10$ ) and quantified the extent of heterogeneity with the 12 statistic. It is common practice to interpret $12>50 \%$ as representing substantial inconsistency or significant statistical heterogeneity. The pooled odds ratio (OR) and $95 \%$ confidence interval $(\mathrm{Cl})$ were calculated to express binary outcome results, while weighted mean difference (WMD) and $95 \% \mathrm{Cl}$ were used for the expression of continuous outcome results.

Subgroup analysis: It is common practice to interpret $12>50 \%$ as representing substantial inconsistency or significant statistical heterogeneity. If this happens, a subgroup analysis may be performed.

Sensitivity analysis: The sensitivity analysis on the literature included, it was through eliminating one study at a time in the process of meta-analysis to observe the change of merging effect, so as to illustrate the stability and accuracy of the results. The publication bias was identified by the funnel plots.

Country(ies) involved: China.

Keywords: Methicillin-resistant Staphylococcus aureus; bloodstream infection; risk factors; mortality.

Contributions of each author:

Author 1 - Mohan Ju.

Author 2 - Yueying Huang.

Author 3 - Xiaofeng Xu.

Author 4 - Yiyi Qian.

Author 5 - Yingmin Bi.

Author 6 - Shuang Liu.

Author 7 - Xiangyu Li.

Author 8 - Shuaiyue Dong.

Author 9 - Jinyi Yuan.

Author 10 - Dongfang Lin. 